\title{
Human Pathologic Validation of Left Ventricular Linear Lesion Formation Guided by Noncontact Mapping
}

\author{
BRADLEY P. KNIGHT, M.D., PAUL BOGNER, M.D.,* KRISTINA WASMER, M.D., \\ and FRED MORADY, M.D.
}

From the Division of Cardiology, Department of Internal Medicine, and *Department of Pathology, University of Michigan Medical Center, Ann Arbor, Michigan

\begin{abstract}
Linear Lesions Guided by Noncontact Mapping. This case report describes the histopathologic findings associated with two left ventricular, linear radiofrequency lesions in a patient who underwent cardiac transplantation shortly after an ablation procedure for ventricular tachycardia. The lesions were created with conventional ablation equipment guided by a noncontact mapping system. The findings provide pathologic validation that continuous, linear lesions are feasible using a noncontact mapping system for guidance. (J Cardiovasc Electrophysiol, Vol. 13, pp. 79-82, January 2002)
\end{abstract}

noncontact mapping, pathology, radiofrequency catheter ablation, ventricular tachycardia

\section{Introduction}

The reentrant circuit during monomorphic ventricular tachycardia (VT) in patients with coronary artery disease usually involves a critical endocardial isthmus that can be targeted with catheter ablation. ${ }^{1,2}$ Occasionally, the isthmus is broad and a linear lesion is required for successful ablation. ${ }^{3-5}$ Because fluoroscopy provides poor resolution during intracardiac catheter manipulation, creation of linear lesions with contiguous applications of radiofrequency energy is difficult. We describe the pathologic findings associated with two endocardial, linear lesions created in a patient with ischemic VT using the nonfluoroscopic guidance of the EnSite $3000^{\mathrm{TM}}$ mapping system (Endocardial Solutions, Inc., St. Paul, MN, USA $)^{6,7}$ and conventional ablation equipment.

\section{Case Report}

A 57-year-old man with coronary artery disease, left ventricular ejection fraction of 0.15 , and congestive heart failure received multiple shocks from his implantable defibrillator. He had undergone three previous catheter ablation procedures for recurrent VT. The stored intracardiac electrograms demonstrated VT at 130 to 135 beats/min.

The patient underwent a catheter ablation procedure 5 days after admission. Intravenous amiodarone and oral mexiletine were discontinued the night before the procedure. A retrograde aortic approach was used to position an EnSite balloon catheter and a quadripolar Blazer ${ }^{\mathrm{TM}}$ ablation catheter with a 4-mm distal electrode (EP Technologies Inc., San Jose, CA, USA) into the left ventricle. Multiple VTs were induced by programmed stimulation. The VTs were associated with hemodynamic collapse and were difficult to pace-terminate. Following several internal cardioversions, the patient had spontaneous monomorphic VT that was associated with a systolic blood pressure of $100 \mathrm{mmHg}$. The VT had a left bundle branch

This manuscript was processed by a guest editor.

Address for correspondence: Bradley P. Knight, M.D., Division of Cardiology, Department of Internal Medicine, University of Michigan Medical Center, 1500 East Medical Center Drive, Ann Arbor, MI 48109. Fax: 734-936-7026; E-mail: bpk@umich.edu

Manuscript received 28 June 2001; Accepted for publication 16 July 2001. block, inferior-axis morphology, and cycle length of 660 msec. This VT was targeted for ablation.

The noncontact mapping system was used to construct virtual, endocardial, isopotential maps of the spontaneous VT using a high-pass filter of $8 \mathrm{~Hz}$ with a color gain between 0.000 and $-0.090 \mathrm{mV}$ (Fig. 1). A line was drawn on the isopotential map that transected the presumed isthmus of the VT reentrant circuit. The locator signal of the EnSite system was used to guide navigation of the ablation catheter. Thirteen contiguous radiofrequency current applications were delivered to the left ventricular posterior septum to create a linear lesion that transected the isthmus using a standard radiofrequency generator (EP Technologies). Current was delivered at each site for 60 seconds using automatic temperature control with a maximum power setting of $50 \mathrm{~W}$ and a target temperature of $65^{\circ}$ to $70^{\circ} \mathrm{C}$. After creation of the linear lesion, the targeted VT was no longer inducible.

Programmed stimulation resulted in induction of VT with a cycle length of 400 msec that was not hemodynamically tolerated. The presystolic zone of the reentrant circuit was identified using the isopotential maps (Fig. 2). Using the same technique that was used to create the first linear lesion, 19 contiguous radiofrequency energy applications were delivered to the anterior left ventricular septum, approximately parallel to the previous line. After the second linear lesion, this VT also was no longer inducible.

The patient developed progressive heart failure and underwent implantation of a left ventricular assist device 5 days after the ablation procedure. Ten days after the ablation procedure, he underwent successful cardiac transplantation. The heart was examined following cardiac transplantation.

Inspection of the endocardium revealed two linear lesions along the left ventricular septum (Fig. 3). Each lesion appeared grossly as a linear, tan discoloration with a surrounding dark, ecchymotic border. The individual lesions were contiguous or overlapping, and the width of each linear lesion ranged from 8 to $14 \mathrm{~mm}$. The more posterior of the two lesions continued beyond where the heart had been transected, but the visible portion had an irregular border, was along a groove, was at least $34 \mathrm{~mm}$ long, and was $8 \mathrm{~mm}$ deep at the base of the septum (Fig. 4). The more anterior of the two lesions had straight edges along the endocardium, was located on a convex portion of the septum, was $27 \mathrm{~mm}$ long, and was $6 \mathrm{~mm}$ deep at the base of the septum. In addition to the linear lesions, there were spotty lesions along the septum and posterior wall believed to 


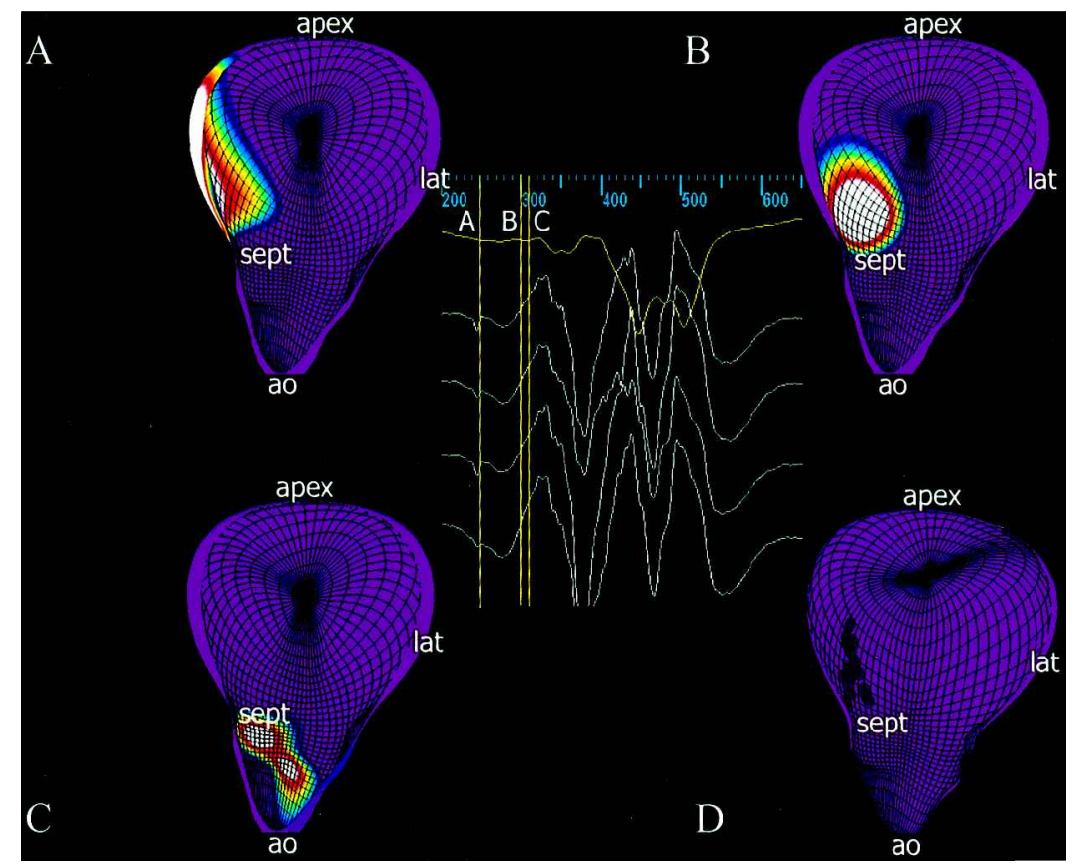

Figure 1. Sequential isopotential maps of the first ventricular tachycardia (VT) that was targeted for ablation. Each map is displayed on a three-dimensional endocardial reconstruction with the posterior wall cut away to view the anterior and septal endocardium, and is oriented with the apex at the top of the picture, aortic (ao) outflow at the bottom, septum (sept) to the left, and lateral (lat) wall to the right. The virtual electrograms recorded during VT from the area of the first ablation line are displayed in the center. Each vertical line represents the time at which the isopotential map was recorded in A through $C$. (A) Isopotential map of VT at a time of $80 \mathrm{msec}$ before the onset of QRS. (B) Isopotential map of VT at a time of $33 \mathrm{msec}$ before the onset of QRS. (C) Isopotential map of VT at a time of $20 \mathrm{msec}$ before the onset of QRS. (D) The linear lesion that was created for the first VT is shown and was created to transect the presystolic conduction path. Each radiofrequency lesion that was delivered to achieve a line of block is denoted with a magenta circle.

represent chronic lesions from previous catheter ablation procedures.

Microscopic analysis of the two linear lesions revealed confluent coagulation necrosis with hemorrhage. There was no normal myocardium within the necrotic areas.

\section{Discussion}

The patient underwent an ablation procedure for VT in which an attempt was made to connect sequential, focal, radiofrequency lesions using the locator signal of a noncontact mapping system, with the goal of creating two linear lesions in the left ventricle. Inspection of the heart following cardiac transplantation revealed continuous, endocardial lesions in the anticipated locations. The accuracy of the locator signal of the noncontact mapping system used in this case has been shown to be within $0.8 \mathrm{~mm}$ in a saline-filled tank simulation ${ }^{8}$ and $1.0 \mathrm{~mm}$ in the canine atrium. ${ }^{9}$ The present case provides human pathologic evidence that continuous linear lesions are feasible in the left ventricle using conventional radiofrequency ablation guided by the locator signal of a noncontact mapping system.

There are two previous reports of the anatomic and histologic characteristics of radiofrequency lesions in the

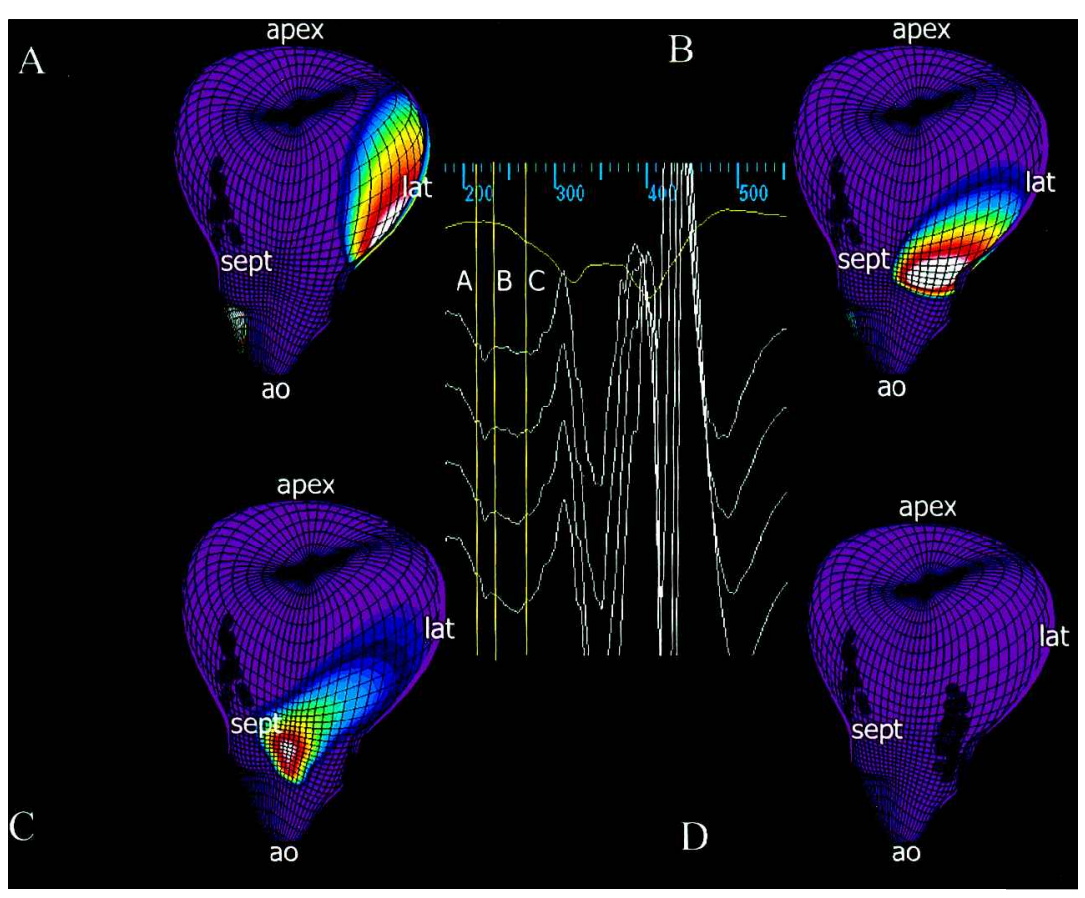

Figure 2. Sequential isopotential maps of the second ventricular tachycardia (VT) that was targeted for ablation. The orientation and abbreviations are the same as in Figure 1. The virtual electrograms recorded during VT from the area of the second ablation line are displayed in the center. Each vertical line represents the time at which the isopotential map was recorded in A through C. (A) Isopotential map of VT at a time of $35 \mathrm{msec}$ before the onset of QRS. (B) Isopotential map of VT at a time of 20 msec before the onset of QRS. (C) Isopotential map of VT at a time of 0 msec before the onset of QRS. (D) Both linear lesions that were created in this patient are shown. The lesion on the right was the second lesion created. Each radiofrequency lesion that was delivered to achieve a line of block is denoted with a magenta circle. 
Figure 3. The heart has been transected basally at the level of the aortic root (Ao) and the posterior wall (POST) has been cut from the base to the apex. The heart is opened to view the endocardium and is oriented with the apex at the top of the picture, base at the bottom, septum (SEPT) to the left, lateral wall (LAT) to the right, and anterior wall (ANT) in the center. A centimeter ruler is positioned at the bottom of the picture for scale. A portion of the hose from the left ventricular assist device (LVAD) is sewn to the apex of the heart and is cut open along the posterior aspect. Two linear endomyocardial lesions are visible along the left ventricular septum (dotted lines). The septum is cut at the level of the basal aspect of each lesion to permit better visualization of the deeper portions of the lesions. Each lesion is continuous, and the width of each lesion ranges from 8 to $13 \mathrm{~mm}$. The more posterior of the two lesions is on the left and continues beyond where the heart had been transected, but the visible portion had an irregular border, is along the groove between the septum and posterior wall, and is at least $34 \mathrm{~mm}$ long. The more anterior of the two lesions is on the right, has straight edges, is located on a convex portion of the septum, and is $27 \mathrm{~mm}$ long. The location of the lesions correlates with the location of the lesion sets that were marked on the virtual endocardium displayed by the noncontact mapping system. Because the heart is cut and opened, the orientation of the heart in this figure is not the same as the orientation of the endocardial reconstruction of the mapping system. There are endocardial scars (*) anteriorly that most likely represent lesions from previous catheter ablation procedures.

left ventricle in humans. ${ }^{10,11}$ The first report described focal lesions using a standard radiofrequency ablation catheter. Lesions ranged from 2 to $10 \mathrm{~mm}$ in width and up to $3 \mathrm{~mm}$ in depth. The second report described lesions created using a saline-cooled radiofrequency catheter. The lesion depth was up to $7 \mathrm{~mm}$. The only human pathologic report of a linear endocardial lesion is a histopathologic description of a linear endocardial scar after radiofrequency ablation of the low, right atrial isthmus for atrial flutter in a patient who underwent cardiac transplantation 4 months later. $^{12}$

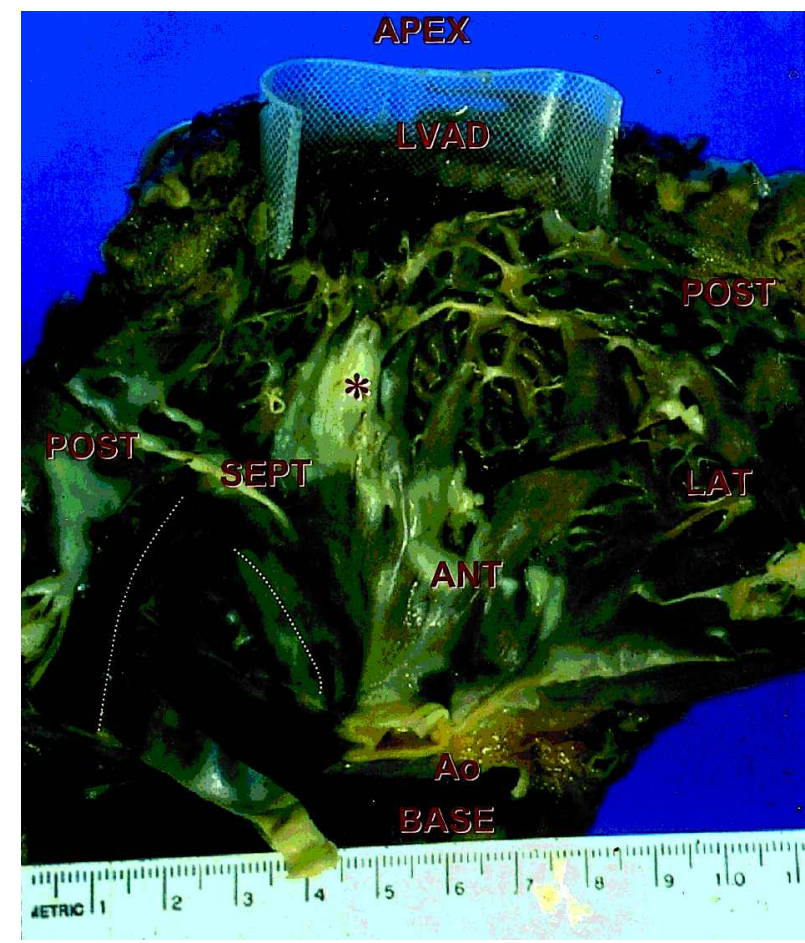

Thomas et al. ${ }^{13}$ used a hand-held device and radiofrequency current to create linear, endocardial, atrial lesions intraoperatively in patients with atrial fibrillation. Despite direct visualization at open heart surgery, discontinuities in the linear lesions were common and resulted in postoperative atrial flutter. The ventricular lesions in the present case were not transmural. However, microscopic analysis of the endomyocardial radiofrequency lesions in this case demonstrated confluent necrosis without viable myocardium within the lesions.

In summary, the present case provides pathologic vali-
Figure 4. Cross-section of the more posterior septal lesion (arrow) is shown in this figure viewed from the base of the heart. The ruler on the right demonstrates that the depth of the lesion at this level is $8 \mathrm{~mm}$ and the width is $14 \mathrm{~mm}$. Abbreviations as in Figure 3.

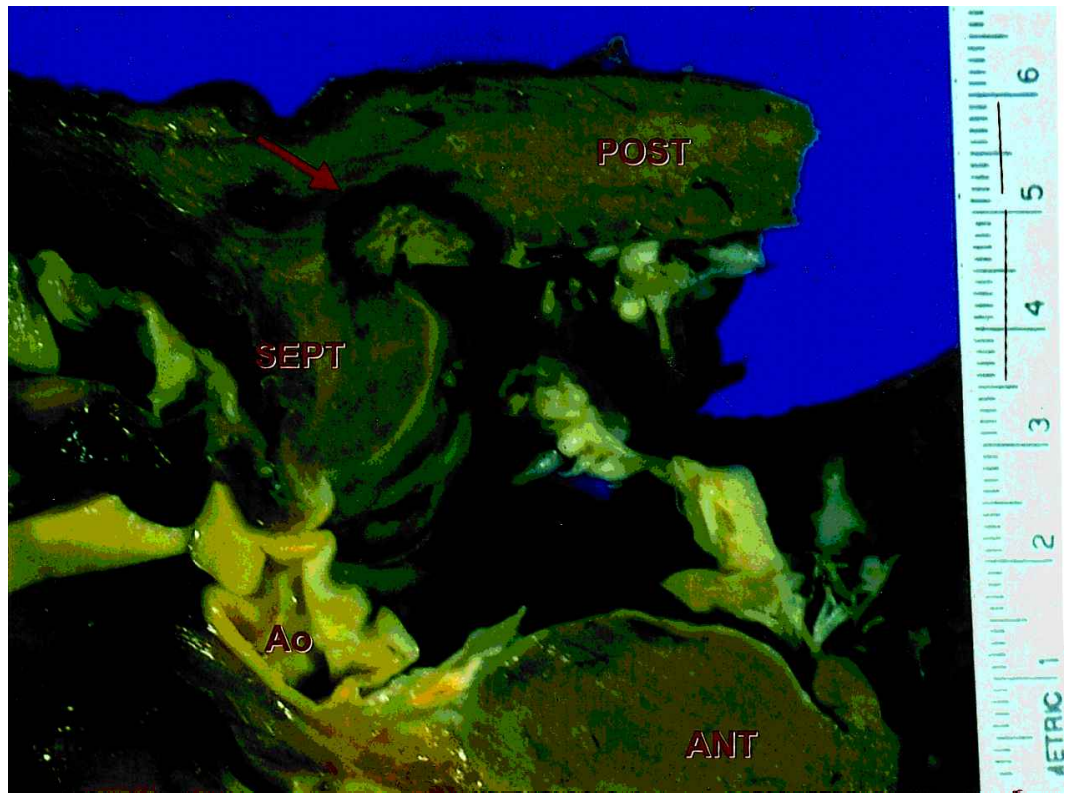


dation that continuous linear lesions are feasible using conventional radiofrequency ablation and a noncontact mapping system.

\section{References}

1. Morady F, Harvey M, Kalbfleisch SJ, El-Atassi R, Calkins H, Langberg JJ: Radiofrequency catheter ablation of VT in patients with coronary artery disease. Circulation 1993;87:363-372.

2. Wilber DJ, Kopp DE, Glascock DN, Kinder CA, Kall JG: Catheter ablation of the mitral isthmus for ventricular tachycardia associated with inferior infarction. Circulation 1995;92:3481-3489.

3. Friedman PA, Packer DL, Hammill SC: Catheter ablation of mitral isthmus ventricular tachycardia using electroanatomically guided linear lesions. J Cardiovasc Electrophysiol 2000;11:466-471.

4. Horton RP, Canby RC, Kessler DJ, Joglar JA, Hume A, Jessen ME, Scott WP, Page RL: Ablation of ventricular tachycardia associated with tetralogy of Fallot: Demonstration of bidirectional block. J Cardiovasc Electrophysiol 1997;8:432-435.

5. Marchlinski FE, Callans DJ, Gottlieb CD, Zado E: Linear ablation lesions for control of unmappable ventricular tachycardia in patients with ischemic and nonischemic cardiomyopathy. Circulation 2000; 101:1288-1296.

6. Strickberger SA, Knight BP, Man KC, Goyal RG, Pelosi F, Flemming M, Hasse C, Morady F: Mapping and ablation of ventricular tachycardia guided by virtual electrograms using a noncontact, computerized mapping system. J Am Coll Cardiol 2000;35:414-421.
7. Schilling RJ, Peters NS, Davies DW: Simultaneous endocardial mapping in the left ventricle using a noncontact catheter. Comparison of contact and reconstructed electrograms during sinus rhythm. Circulation 1998;98:887-898.

8. Gornick CC, Adler SW, Pederson B, Hauck J, Budd J, Schweitzer J: Validation of a new noncontact catheter system for electroanatomic mapping of left ventricular endocardium. Circulation 1999;99:829-835.

9. Kadish A, Hauck J, Pederson B, Beatty G, Gornick: Mapping of atrial activation with a noncontact, multielectrode catheter in dogs. Circulation 1999;99:1906-1913.

10. Bartlett TG, Mitchell R, Friedman PL, Stevenson WG: Histologic evolution of radiofrequency lesions in an old human myocardial infarct causing ventricular tachycardia. J Cardiovasc Electrophysiol 1995;6: 625-629.

11. Delacretaz E, Stevenson WG, Winters GL, Mitchell RN, Stewart S, Lynch K, Friedman PL: Ablation of ventricular tachycardia with a saline-cooled radiofrequency catheter: Anatomic and histologic characteristics of the lesions in humans. J Cardiovasc Electrophysiol 1999; 10:860-865

12. Leonelli FM, Natale A, O'Connor W: Human histopathologic findings following radiofrequency ablation of the tricuspid-inferior vena cava isthmus. J Cardiovasc Electrophysiol 1999;10:599-602.

13. Thomas SP, Nunn GR, Nicholson IA, Rees A, Daly MP, Chard RB, Ross DL: Mechanism, localization and cure of atrial arrhythmias occurring after a new intraoperative endocardial radiofrequency ablation procedure for atrial fibrillation. J Am Coll Cardiol 2000;35:442450 